\title{
von Hauff, Luba (2020): China, the West, and \\ Democratization. The Struggle for the Local and the Global in Post-Soviet Kazakhstan
}

\author{
Abingdon/New York: Routledge. 226 Seiten. $£ 120.00$
}

\section{Alexander Brand}

Online publiziert: 26. Oktober 2020

(C) Der/die Autor(en) 2020

Die internationalen Beziehungen erleben derzeit eine Phase dramatischer Umbrüche. $\mathrm{Zu}$ den bedeutendsten Phänomenen zählen die aus dem Aufstieg Chinas sowie dem augenscheinlichen Rückzug der USA aus einer Führungsrolle sich ergebenden Konsequenzen. Erleben wir eine dauerhafte Erosion demokratischer Spielregeln? Ist die Welle der Demokratisierung verebbt? Welche Rolle spielen ,alternative“, maßgeblich von China ins Leben gerufene Institutionen wie etwa die Shanghaier Organisation für Zusammenarbeit (SOZ) dabei? Sind sie auf Stabilisierung, gar auf einen Export von Autokratie hin ausgerichtet und damit Teil einer absichtsvollen chinesischen Grand Strategy? Wandel manifestiert sich jedoch nicht allein global, auch viele Regionen sind in Bewegung geraten. $\mathrm{Zu}$ denjenigen mit dem größten Potenzial für anstehende Umbrüche dürfte das hierzulande, und leider auch in den hiesigen Internationalen Beziehungen, bis dato eher vernachlässigte Zentralasien zählen. Dabei kommt Zentralasien eine Brückenfunktion zwischen „dem Westen“ und Asien, Russland und nicht zuletzt auch China zu. Maßgeblich aus diesem Grund begreift auch die jüngst von der EU beschlossene neue Zentralasienstrategie die Region gerade nicht als peripher zum Weltgeschehen. In welche Richtung aber wird sich Zentralasien bewegen?

In ihrer 2017 vorgelegten und 2020 in überarbeiteter Form veröffentlichten Dissertationsschrift widmet sich Luba von Hauff eben diesen spannenden wie globalpolitisch bedeutsamen Fragen. Im Mittelpunkt ihres Interesses stehen Kasachstan und dessen Transformationsdynamiken seit der Unabhängigkeit 1991. Dieser Fall ist gut gewählt, da er erlaubt, die genannten Erkenntnisinteressen nicht allein separat, sondern in deren historischer wie kausaler Verknüpfung zu diskutieren.

\footnotetext{
A. Brand $(\bowtie)$

Hochschule Rhein-Waal, Kleve, Deutschland

E-Mail: alexander.brand@hochschule-rhein-waal.de
} 
Von Hauff stellt in ihrer Untersuchung zwei Fragen zentral: Positioniert sich China auch ideologisch als strategischer Wettbewerber zum „Westen“, indem es intentional dessen auf Demokratisierung hin ausgerichtetes Normsetzungsmonopol zu untergraben versucht? Und wie lässt sich Kasachstans Transformationspfad in einem sich wandelnden Koordinatensystem verorten? Hat es, in Anlehnung an globale Machtverschiebungen, eine „,normative Kehrtwende“ (S. 5) vollzogen?

Das konzeptionelle Rückgrat des Buches bildet Kapitel 2 zum Thema „Sozialisation", also der Einbindung von Staaten in Wertesysteme und die sukzessive Übernahme normativer Handlungsvorgaben durch diese. Vor allem geht es von Hauff darum herauszuarbeiten, wie der Erfolg bzw. Misserfolg von Sozialisation zu erfassen und bewerten ist. Die Idee, Staaten als Mitglieder konkurrierender sozialer Gruppen (i. S. von Wertegemeinschaften) auszuweisen, erlaubt es zu eruieren, inwieweit ein Akteur den ideologischen Vorgaben der je relevanten Führungsmacht folgt. Über die Sozialisationstiefe entscheiden allerdings vor allem auch die von der Autorin in der Debatte stark gemachten lokalen Gegebenheiten bzw. kognitiven Prägungen (S. 49-52 und 78-81). Im Falle Kasachstans zählen zu diesen Prägungen nicht zuletzt die traditionelle Orientierung hin auf patrimoniale Herrschaftsstrukturen sowie eine ausgeprägte Kultur der Patronage und Kooptation als Erbe aus Sowjetzeiten.

Der Hauptteil des Buches diskutiert in jeweils zwei Kapiteln den Weg Kasachstans seit 1991 sowie die Rolle Chinas in Zentralasien, letzteres zum einen mit Blick auf die Gründung und Etablierung der SOZ und zum anderen, mit einem Perspektivenschwenk in die Zukunft, hinsichtlich der „One Belt, One Road“-Strategie. Mindestens zwei unterschiedliche Lesarten der Studie lassen sich dabei identifizieren. Erstens beschreibt von Hauff plausibel, wie chinesische Regionalpolitik Impulse für eine Ent-Demokratisierung zu setzen vermag. Und in der Tat bietet es sich an, hier den „Geist von Shanghai“ im Kontext der SOZ als zentralen Referenzpunkt zu diskutieren, auch wenn Chinas Rolle als ,,spoiler“ (S. 5) im vorliegenden Buch eher geschlussfolgert als nachgewiesen wird. Zweitens legt die Autorin eine detailreiche Schilderung innenpolitischer und -gesellschaftlicher Entwicklungen in Kasachstan seit 1991 vor. Vor allem verdeutlicht sie die Kluft zwischen deklaratorischer Unterstützung von Demokratie und den alltäglichen Praxen von Kooptation, tendenziell eher sanfter Repression und Lenkung eines selektiven Pluralismus von oben. Bereits während der Lektüre stellt sich so die Frage, ob es denn eigentlich jemals einen authentischen Demokratisierungsimpuls in Kasachstan gegeben hat.

Oder pointierter: Legt die gewählte Sozialisierungsperspektive nahe, es habe sich um ein Oszillieren der kasachischen Führung zwischen zwei normativen Welten Demokratie und ein asiatischer bzw. kasachischer Weg des kaschierten Autoritarismus - gehandelt, so vermittelt die geschilderte empirische Evidenz in den Kapiteln 3 und 4 einen eindeutigeren Eindruck. Sollte es wirklich eine normative Kehrtwende gegeben haben, so dürfte die in den 1990er-Jahren erfolgte rhetorische Annäherung an den Westen und die OSZE nicht rein taktisch-opportunistischer Natur gewesen sein. Legt man aber die von der Autorin auf fünf Seiten konzise zusammengefassten Kriterien für eine authentische Pluralisierung (S. 10-15) an, geraten schon für die erste Dekade der kasachischen Unabhängigkeit viel blumige Rhetorik, bisweilen im orwellschen Gewand, und nicht wenige Potemkinsche Dörfer ins Blickfeld. Mit 
Ernsthaftigkeit betrieben wurde Demokratisierung selten (und schon gar nicht „,von oben" herab), Tiefe erreichte sie kaum.

Unter dem Firnis pseudo-demokratischer Prozeduren lugen Klientelismus, Oligarchenzwist und autoritäre Lenkung durch den Präsidenten allzu oft hervor. Die Schilderungen von Hauffs dazu sind aufschlussreich und ebenso ernüchternd. Wenn aber die Hinwendung zu ,westlichen Werten“ nie authentisch war, wie groß ist dann der Umschwung, ob nun von China wohlwollend begleitet oder willentlich herbeigeführt? Zudem lässt das Auftreten erster autokratischer Rückschritte bereits Mitte der 1990er-Jahre, wie von Hauff selbst mehrfach betont (u.a. S. 85-94), Zweifel daran aufkommen, dass China und die SOZ hier eine substanzielle Trendumkehr bewirkt haben. Vielmehr scheint deren Engagement in Zentralasien allenfalls die Notwendigkeit für die kasachische Führung, weiterhin demokratische Mimikry zu betreiben, gelockert zu haben.

Es ist das große Verdienst der Autorin, diese Themenkomplexe in ihrer wechselseitigen Verschränkung für ein akademisches Publikum wie für einen breiteren Kreis von Leserinnen und Lesern zu erschließen. Während Kapitel 2 an Fachdebatten andockt, bieten die übrigen Teile des Buches all jenen, die sich vor allem für die Region Zentralasien, globale Machtverschiebungen und die Zukunft der Demokratie interessieren, einen hervorragenden Diskussionsansatz. Dies hat nicht zuletzt damit zu tun, dass von Hauff einen angenehmen, gleichzeitig gehaltvollen und zugänglichen Sprachstil pflegt. Es bleibt zu hoffen, dass auch und gerade die deutsche Debatte ihre Monografie breitestmöglich rezipiert. Die zentralen in ihr verhandelten Fragen jedenfalls werden die internationalen Beziehungen in den kommenden Jahren entscheidend mitprägen.

Funding Open Access funding enabled and organized by Projekt DEAL

Open Access Dieser Artikel wird unter der Creative Commons Namensnennung 4.0 International Lizenz veröffentlicht, welche die Nutzung, Vervielfältigung, Bearbeitung, Verbreitung und Wiedergabe in jeglichem Medium und Format erlaubt, sofern Sie den/die ursprünglichen Autor(en) und die Quelle ordnungsgemäß nennen, einen Link zur Creative Commons Lizenz beifügen und angeben, ob Änderungen vorgenommen wurden.

Die in diesem Artikel enthaltenen Bilder und sonstiges Drittmaterial unterliegen ebenfalls der genannten Creative Commons Lizenz, sofern sich aus der Abbildungslegende nichts anderes ergibt. Sofern das betreffende Material nicht unter der genannten Creative Commons Lizenz steht und die betreffende Handlung nicht nach gesetzlichen Vorschriften erlaubt ist, ist für die oben aufgeführten Weiterverwendungen des Materials die Einwilligung des jeweiligen Rechteinhabers einzuholen.

Weitere Details zur Lizenz entnehmen Sie bitte der Lizenzinformation auf http://creativecommons.org/ licenses/by/4.0/deed.de. 